\title{
Aligning New Approaches to Accelerate the Development of Non-opioid Analgesic Therapies
}

\author{
Christine N. Sang ${ }^{1}$ (D) $\cdot$ William K. Schmidt ${ }^{2}$
}

Accepted: 21 September 2020 / Published online: 14 October 2020

(C) The American Society for Experimental NeuroTherapeutics, Inc. 2020

The opioid epidemic has transformed pain research, not only in the sense of urgency with which analgesics are being developed, but also in the way in which hypotheses are being tested. This paradigm shift requires a look at both preclinical and clinical data with a perspective that follows traditional pharmaceutical approaches while incorporating new technologies. Moreover, with the launch of the National Institutes of Health (NIH) Helping to End Addiction Long-term (HEAL) Initiative in April 2018, the collaborative effort with the US Food and Drug Administration (FDA) and NIH has become ever more important.

In the previous Neurotherapeutics Special Issue on Pain (2009), our focus was on the advances in the field of neuropathic pain with an emphasis on the successful drug development program of gabapentin as the model for analgesic drug development. The treatments were almost entirely small molecules and the innumerable potential factors that contributed to failures to achieve clinically meaningful analgesic effects were focused on issues such as the ability of the compound to reach the target site. In 2020, many of the tools and technologies that have facilitated the discovery of analgesic targets and analgesic drug development were adapted from other therapeutic indications. These include the development of monoclonal antibodies to transmembrane protein 119 that enable immunostaining of microglia [1]; advances in transcriptomics [2, 3]; the development of subtype-selective small molecule radioligands [4]; and the improvement of viral and nonviral vectors and gene editing technologies [5].

Christine N. Sang

csang@bwh.harvard.edu

1 Translational Pain Research, Brigham and Women's Hospital, Harvard Medical School, 75 Francis Street, Boston, MA 02115, USA

2 NorthStar Consulting LLC, Davis, CA, USA
Many of these innovations have revolutionized the landscape of new analgesics entering clinical trials. Table 1 presents a summary of 214 tracked compounds in development (as of December 31, 2019) that we have been following for the past 3 years; Table 2 presents the data by primary indication. The tables are extracted from a larger database that is available for download on www.paintrials.org/analgdrugdevt. All of the entries are based on publicly available data that are referenced by hyperlinks within the table. These include 179 compounds in active clinical development, 8 compounds that were recently approved (2018), and 27 compounds that were discontinued or where no development has been reported for extended periods of time. It currently takes, on average, 12.8 years to develop new therapeutics; for orphan drugs, it can take 2.3 years longer [6]. Central nervous system (CNS) drugs have even higher failure rates [7]. Even then, the road to an approved new drug is uncertain. Wong et al. [8] evaluated the probability of successful development for 21,000 unique drugs from January 2000 through October 2015; only 15\% of CNS drugs entering phase 1 clinical trials received FDA approval.

Clearly, innovations are needed to accelerate the development of novel, more effective, and less addictive analgesic compounds with fewer adverse effects than the commercially available drugs in use today. These include better preclinical and clinical methods with predictive and prognostic biomarkers to develop drugs with a higher chance of success in gaining approval. Indeed, Dr. Francis Collins, Director of the NIH, has challenged the industry to discover improved methods for analgesic drug development and to reduce the development time for new drugs by $50 \%$ in order to address the opioid crisis in the United States. The NIH committed up to $\$ 1$ billion to this effort in April 2018 to increase the number of novel compounds going into the pipeline and improve their chances of success in gaining FDA approval.

With these considerations in mind, this special Neurotherapeutics issue includes the insights of representatives from the FDA, academia, industry, and the NIH. This 
Table 1 Summary of analgesic drugs in development by therapeutic target (December 31, 2019)

\begin{tabular}{|c|c|c|c|c|c|c|}
\hline Therapeutic target & Phase 1 & Phase 2 & Phase 3 & $\begin{array}{l}\text { NDA/BLA } \\
\text { submitted }\end{array}$ & $\begin{array}{l}\text { NDA/BLA } \\
\text { approved }\end{array}$ & $\begin{array}{l}\text { Recently } \\
\text { discontinued }\end{array}$ \\
\hline$\alpha 2 \delta-1$ & & 1 & 1 & & & \\
\hline $5-\mathrm{HT}_{2}$ antagonist & & 1 & & & & \\
\hline Alpha $_{2}$ agonist & & 1 & & & & \\
\hline AMPA glutamate & & 1 & & & & \\
\hline COMBINATION products & 2 & 8 & 5 & 3 & & 2 \\
\hline BIOLOGICS* & 15 & 23 & 13 & & 2 & 8 \\
\hline Bisphosphonate & & & 2 & & & 1 \\
\hline Cannabinoid (CB1, CB2) & 3 & 2 & & & & \\
\hline Ion channel: calcium ( $\mathrm{CaV}$ channel) & & & 1 & & & \\
\hline Ion channel: sodium ( $\mathrm{NaV}$ channel) & & 2 & & & & 1 \\
\hline Ion channel: potassium (KV channel) & & 1 & & & & \\
\hline CCR2 cytokine antagonist & 1 & & & & & \\
\hline CGRP antagonist & & 2 & 1 & 2 & 3 & \\
\hline Corticosteroid & & & 4 & & & \\
\hline Opioid: mu agonist & 3 & & 5 & 3 & & 7 \\
\hline Opioid: kappa agonist & & & 1 & & & \\
\hline Opioid: delta agonist & 1 & & & & & \\
\hline Opioid: endomorphin & 1 & & & & & \\
\hline Opioid: enkephalinase inhibitor & 1 & & & & & \\
\hline Opioid: other (NOP, undisclosed) & & 1 & 1 & & & \\
\hline Opioid: prodrug & 2 & & & & & 2 \\
\hline GnRH antagonist & & & 1 & & & \\
\hline Ergot alkaloid & & & 2 & & & \\
\hline Imidazoline agonist (I2) & & 1 & & & & \\
\hline JAK1, pan-JAK inhibitor & & 1 & & 1 & 1 & \\
\hline Local anesthetic & 2 & 2 & 1 & 1 & & \\
\hline Neurostimulation & & 2 & 1 & & & \\
\hline NGF inhibitor & & & 2 & & & \\
\hline NMDA antagonist & & 2 & & & & 1 \\
\hline NSAID (COX-1, COX-2) & 2 & 2 & 1 & 1 & & \\
\hline PDE9 inhibitor & & 1 & & & & \\
\hline \multicolumn{7}{|l|}{ Prostaglandin synthase inhibitor } \\
\hline Sigma channel blocker & & 1 & & & & \\
\hline Soluble epoxide hydrolase $(\mathrm{sEH})$ inhibitor & 1 & & & & & \\
\hline Soluble guanylate cyclase (sGC) & & 1 & & & & \\
\hline Somatostatin SSTR4 agonist & 1 & & & & & \\
\hline Superoxide dismutase mimic & & 1 & & & & \\
\hline Syk inhibitor & & 1 & & & & \\
\hline Triptan & & 1 & & 1 & & \\
\hline TrkA inhibitor & 1 & 1 & & & & 1 \\
\hline TRPA1 antagonist & & 1 & & & & \\
\hline TRPV1 agonist (capsaicin, resiniferatoxin) & 1 & 2 & 1 & & & \\
\hline Wnt inhibitor & & & 1 & & & \\
\hline Other mechanisms of action & 6 & 9 & 3 & & 2 & 4 \\
\hline Total & 43 & 72 & 47 & 12 & 8 & 27 \\
\hline
\end{tabular}

*BIOLOGICS (unless otherwise included above) include the following: ammonia-oxidizing bacteria (AOB), anti-CCP vaccine (citrullinated peptide dendritic cell immunotherapy), dipeptide aspartyl-alanyl diketopiperazine (DA-DKP), autologous bone morphogenetic protein (BMP-7), autologous gene therapy targeting BCL11A protein, BCG vaccine, chondroitin-sulfate-ABC endolyase, IL-1 inhibitor, IL-6 inhibitor, IL-10 inhibitor, IL-23 inhibitor, innate defense regulator (IDR), innate repair receptor peptide (IRR), gene encoding type VII collagen (COL7), NF-kB decoy, PACAP-38 inhibitor, P-selectin inhibitor, PEGylated uric acid specific enzyme, plasmid gene therapy encoding hepatocyte growth factor (HGF), proteoglycan aggrecan mimetic, stem cells, transthyretin (TTR) protein, type VII gene replacement

issue is organized into four sections, ranging from clinical outcome assessments, to approaches to modulating peripheral and central nervous system targets, to opportunities for federal funding that are reserved for analgesic drug development.

Section I: Emerging Clinical Technologies includes two papers that address the development of clinical outcome assessment endpoints in clinical trials that each consider the complexity of the multidimensional pain experience. St. Clair et al. [9] present an overview of the regulatory considerations when selecting and developing analgesic clinical trial endpoints in specific populations, with an emphasis on formulating measurement strategies that consider the comprehensive view of the patients' pain experience in determining clinical benefit, defined as how a patient feels, functions, or 
Table 2 Summary of analgesic drugs in development by primary indication (December 31, 2019)

\begin{tabular}{lccccc}
\hline Primary indication & Phase 1 & Phase 2 & Phase 3 & $\begin{array}{l}\text { NDA/BLA } \\
\text { submitted }\end{array}$ & $\begin{array}{l}\text { NDA/BLA } \\
\text { approved }\end{array}$ \\
\hline
\end{tabular}

Arthritis: ankylosing spondylitis 1

Arthritis: axial spondyloarthritis

Arthritis: juvenile rheumatoid arthritis (JRA)

Arthritis: osteoarthritis, hip or knee

Arthritis: other

5

Arthritis: rheumatoid arthritis

Bladder pain (BPS/IC)

Cancer pain

Chemotherapy-induced peripheral neuropathy (CIPN)

Chronic low back pain (LBP)

Chronic pain (general)

Chronic pain: Anal fissure

Complex regional pain syndrome (CRPS)

Dermal pain, other

Dermal pain/itch: prurigo nodularis $(\mathrm{PN})$

Dermal: topical

Endometriosis pain

Fibromyalgia

Gout

Headache: cluster

Inflammatory pain, unspecified

Irritable bowel syndrome (IBS)

Migraine: acute or chronic

Migraine: prevention

Musculoskeletal pain

Neuropathic pain, unspecified

Neuropathic: diabetic peripheral neuropathy (DPN)

Neuropathic: postherpetic neuralgia (PHN)

Neuropathic: sciatica

Ocular pain

Opioid respiratory depression

Oral: mucositis

Oral: Sjogren's syndrome

Post-amputation pain

Postoperative pain: acute

Postoperative pain: chronic

Rare disease: epidermolysis bullosa (EB)

Rare disease: familial amyloid polyneuropathy (FAP)

Rare disease: other

Rare disease: pemphigus vulgaris (PV)

Rare disease: sickle cell pain / disease

Ulcerative colitis (Crohn's)

Uremic pruritus

Unspecified pain

Total

8

2

1

1

4

2

1

4

4

2

2

43

3

1

4

1

1

1

1

8

3

72

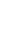

1

17

2

1

1

$1 \quad 4$

$1 \quad 1$

1

1

1

5

1

1

1

5

3

1

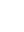

1

6

62

2

1

2

1

5

5

23

1

$1 \quad 1$

1

1

2

1

47

12

8

27 
survives (in the contex of the drug product; the disease state; the patient population; pain measurement that includes not only physical but also emotional and social functioning; and the relevant stakeholders). Goldstein et al. [10] present an approach to evaluating pain and function by using a mobile platform for measuring pain, emotions, and associated bodily feelings. Moreover, they have developed and validated predictive models using artificial intelligence/machine learning tools to demonstrate that the best predictors of future pain in patients with chronic low back pain are not simply pain intensity but the interactions between (1) bodily expressed fatigue with negative affect and (2) positive affect with past pain.

Section II: Approaches to modulating targets presents two reviews of novel peripheral targets for analgesic drug development and six novel CNS targets. Ciotu and Fischer [11] survey an enormous literature of mediators and antagonists acting on peripheral sensory neurons that include some now familiar targets (e.g., substance P, calcitonin gene-related peptide, tumor necrosis factor-alpha) and others that are less familiar and, hence, of interest for developing novel therapeutics (lysophospholipids, trypsin, and a Disintegrin and Metalloproteinase with Thrombospondin motifs (ADAMTS)). Krajewski [12] discusses purinergic P2X3 and $\mathrm{P} 2 \mathrm{X} 2 / 3$ receptors on sensory nerves that are critical for sensory transduction. Clinical studies have shown promise in treatment for bladder pain and pain associated with osteoarthritis. Bannister and Dickenson [13] review supraspinal mechanisms for pain control and discuss the supraspinally mediated analgesic actions of opioidergic, anti-convulsant, and anti-depressant drugs. Donnelly et al. [14] discuss glial cell mechanisms in chronic pain focusing on ways that microglia and astrocytes contribute to chronic pain and point to potentially druggable hemichannels and proteases produced by reactive microglia and astrocytes that may regulate acute or chronic pain. Li et al. [15] discuss adenylyl cyclase subtype $1(\mathrm{AC} 1)$ as a key intracellular protein that causes both presynaptic and postsynaptic forms of long-term potentiation (LTP); inhibiting AC1 may block behavioral sensitization and injuryrelated anxiety in animal models of chronic pain. Hughes and Todd's review [16] focuses on inhibitory neurons in the spinal cord that are critical for setting pain thresholds; they discuss how disinhibition of spinal dorsal horn interneurons can produce aberrant sensory processing associated with chronic pain states. Ji et al. [17] present neuromodulation techniques (e.g., vagal nerve stimulation, auricular electroacupuncture) that may release specialized pro-resolving mediators (SPMs), which are lipid molecules produced during the resolution phase of inflammation. Recent studies suggest that SPMs inhibit inflammatory pain, postoperative pain, neuropathic pain, and cancer pain in rodent models via immune, glial, and neuronal modulations. Wagner et al. [18] discuss epoxy fatty acids (EpFAs) formed as metabolites of membrane lipids that may limit pain and inflammation by controlling endoplasmic reticulum stress and blocking mitochondrial dysfunction. These authors have recently developed a series of small molecule inhibitors of soluble epoxide hydrolase (sEH) that stabilize EpFA in vivo as a new approach for reducing acute and chronic pain.

In Section III: Additional insights into peripheral and central nervous system mechanisms, Song et al. [19] review the interactions between and among various small non-coding RNA species (including lncRNAs, circRNAs, and miRNAs) and their ability to regulate protein expression from mRNA in neuropathic pain.

In Section IV: The Future, Iyengar et al. [20] present the NIH HEAL Initiative, with the goal of accelerating the discovery and development of non-addictive pain treatments. This issue also includes three Original Articles that show the use of specific strategies to reduce hyperalgesia in rodent models of neuropathic pain [21,22] and the development of analgesic tolerance to the chronic use of MK-801 resulting from the sensitization of presynaptic N-methyl-D-aspartate receptors and associated glutamate release [23]. Additionally, this issue includes articles and commentaries that address strategies for regeneration and restoration in neurodegenerative disorders; we view these papers as opportunities for potential cross-fertilization.

Tremendous progress in recent years in the understanding of pain targets has led to the development of over $200 \mathrm{com}$ pounds for acute and chronic pain, but the approval of new analgesics has been disappointing. We look forward to seeing at least some of these advances realized to bring new analgesics to the patients. This would require that new approaches to preclinical and clinical drug discovery be aligned in order to accelerate the development of non-opioid analgesic therapies.

Required Author Forms Disclosure forms provided by the authors are available with the online version of this article.

\section{References}

1. Bennett ML, Bennett FC, Liddelow SA, et al. New tools for studying microglia in the mouse and human CNS. Proc Natl Acad Sci U S A. 2016;113(12):E1738-E1746. https://doi.org/10.1073/pnas. 1525528113

2. Ray P, Torck A, Quigley L, Wangzhou A, Neiman M, Rao C, Lam T, Kim JY, Kim TH, Zhang MQ, Dussor G, Price TJ. Comparative transcriptome profiling of the human and mouse dorsal root ganglia: an RNA-seq-based resource for pain and sensory neuroscience research. PAIN. 2018;159(7):1325-1345. https://doi.org/10. 1097/j.pain.0000000000001217

3. Wangzhou A, Mcllvried LA, Paige C, Barragan-Iglesias P, Shiers S, Ahmad A, Guzman CA, Dussor G, Ray PR, Gereau RW 4th, Price TJ. Pharmacological target-focused transcriptomic analysis of native vs cultured human and mouse dorsal root ganglia. PAIN. 
2020;161(7):1497-1517. https://doi.org/10.1097/j.pain. 0000000000001866

4. Maier DL, Hill G, Ding M, et al. Pre-clinical validation of a novel alpha-7 nicotinic receptor radiotracer, [(3)H]AZ11637326: target localization, biodistribution and ligand occupancy in the rat brain. Neuropharmacology. 2011;61(1-2):161-171. https://doi.org/10. 1016/j.neuropharm.2011.03.024

5. Ingusci S, Verlengia G, Soukupova M, Zucchini S, Simonato M. Gene Therapy Tools for Brain Diseases. Front Pharmacol. 2019;10: 724. https://doi.org/10.3389/fphar.2019.00724

6. Tufts Center for the Study of Drug Development Impact Report. Patent-to-launch time for orphan drugs is 2.3 years longer vs. other drugs. 20(3), May/June 2018.

7. Gribkoff VK, Kaczmarek LK. The need for new approaches in CNS drug discovery: Why drugs have failed, and what can be done to improve outcomes. Neuropharmacology. 2017;120:11-19. https://doi.org/10.1016/j.neuropharm.2016.03.021

8. Wong $\mathrm{CH}$, Siah KW, Lo AW. Estimation of clinical trial success rates and related parameters. Biostatistics. 2019;20(2):273-286. https://doi.org/10.1093/biostatistics/kxx069

9. St. Clair CO, Papadopoulos EJ. Considerations in the Assessment of Clinical Benefit with a Focus on Pain: A Regulatory Perspective. Neurotherapeutics. 2020. https://doi.org/10.1007/s13311-02000906-6

10. Goldstein P, Ashar Y, Tesarz J, Kazgan M, Cetin B, Wager TD. Emerging clinical technology: Application of machine learning to chronic pain assessments based on emotional body maps. Neurotherapeutics. 2020. https://doi.org/10.1007/s13311-02000886-7

11. Ciotu CI, Fischer MJM. Novel analgesics with peripheral targets.

12. Krajewski JL. P2X3-containing receptors as targets for the treatment of chronic pain. Neurotherapeutics. 2020. https://doi.org/10. 1007/s13311-020-00934-2

13. Bannister K, Dickenson AH. Central Nervous System Targets: Supraspinal Mechanisms of Analgesia. Neurotherapeutics. 2020. https://doi.org/10.1007/s13311-020-00887-6

14. Donnelly CR, Andriessen AS, Chen G, Wang K, Jiang C, Maixner W, Ji RR. Central nervous system targets: glial cell mechanisms in chronic pain. Neurotherapeutics. 2020. https://doi.org/10.1007/ s13311-020-00905-7

15. Li XH, Chen QY, Zhuo M. Neuronal adenylyl cyclase targeting central plasticity for the treatment of chronic pain. Neurotherapeutics. 2020. https://doi.org/10.1007/s13311-020-00927-1
16. Hughes DI, Todd AJ. Central nervous system targets: Inhibitory Interneurons in the Spinal Cord. Neurotherapeutics. 2020. https:// doi.org/10.1007/s13311-020-00936-0

17. Tao X, Lee MS, Donnelly CR, Ji RR. Neuromodulation, Specialized Pro-resolving Mediators, and Resolution of Pain. Neurotherapeutics. 2020. https://doi.org/10.1007/s13311-02000892-9

18. Wagner KM, Gomes A, McReynolds CB, Hammock BD. Soluble Epoxide Hydrolase Regulation of Lipid Mediators Limits Pain. Neurotherapeutics. 2020. https://doi.org/10.1007/s13311-02000916-4

19. Song G, Yang Z, Guo J, Zheng Y, Su X, Wang X. Interactions among lncRNA/circRNA, miRNA and mRNA in neuropathic pain. Neurotherapeutics. 2020. https://doi.org/10.1007/s13311-02000881-y

20. Iyengar S, Woller SA, Hommer R, Beierlein J, Wright CB, Tamiz AP, Karp BI. Critical NIH Resources to Advance Therapies for Pain: Preclinical Screening Program and Phase II Human Clinical Trial Network. Neurotherapeutics. 2020. https://doi.org/10.1007/ s13311-020-00918-2

21. Michaeli L, Di Cesare Mannelli L, Del Bello F, Giannella M, Piergentili A, Quaglia W, Carrino D, Pacini A, Ghelardini C. The Use of the Selective Imidazoline I1 Receptor Agonist Carbophenyline as a Strategy for Neuropathic Pain Relief: Preclinical Evaluation in a Mouse Model of Oxaliplatin-Induced Neurotoxicity. Neurotherapeutics. 2020. https://doi.org/10.1007/ s13311-020-00873-y.

22. Hegazy, N., Rezq, S. \& Fahmy, A. Mechanisms Involved in Superiority of Angiotensin Receptor Bockade over ACE Inhibition in Attenuating Neuropathic Pain Induced in Rats. Neurotherapeutics (2020). https://doi.org/10.1007/s13311-02000912-8

23. Li J, Zhang L, Xu C, Lin YH, Zhang Y, Wu HY, Chang L, Zhang YD, Luo CX, Li F, Zhu DY. Prolonged Use of NMDAR Antagonist Develops Analgesic Tolerance in Neuropathic Pain via Nitric Oxide Reduction-Induced GABAergic Disinhibition. Neurotherapeutics. 2020. https://doi.org/10.1007/s13311-02000883-w.

Publisher's Note Springer Nature remains neutral with regard to jurisdictional claims in published maps and institutional affiliations. 